\title{
Theoretical Limits of Speed and Resolution for Kinodynamic Planning in a Poisson Forest
}

\author{
Sanjiban Choudhury*, Sebastian Scherer*, J. Andrew Bagnell* \\ * The Robotics Institute \\ Carnegie Mellon University, 5000 Forbes Avenue, Pittsburgh, PA 15213 \\ Email: (sanjiban, basti)@cmu.edu, dbagnell@ri.cmu.edu
}

\begin{abstract}
The performance of a state lattice motion planning algorithm depends critically on the resolution of the lattice to ensure a balance between solution quality and computation time. There is currently no theoretical basis for selecting the resolution because of its dependence on the robot dynamics and the distribution of obstacles. In this paper, we examine the problem of motion planning on a resolution constrained lattice for a robot with non-linear dynamics operating in an environment with randomly generated disc shaped obstacles sampled from a homogeneous Poisson process. We present a unified framework for computing explicit solutions to two problems - i) the critical planning resolution which guarantees the existence of an infinite collision free trajectory in the search graph ii) the critical speed limit which guarantees infinite collision free motion. In contrast to techniques used by Karaman and Frazzoli [11], we use a novel approach that maps the problem to parameters of directed asymmetric hexagonal lattice bond percolation. Since standard percolation theory offers no results for this lattice, we map the lattice to an infinite absorbing Markov chain and use results pertaining to its survival to obtain bounds on the parameters. As a result, we are able to derive theoretical expressions that relate the non-linear dynamics of a robot, the resolution of the search graph and the density of the Poisson process. We validate the theoretical bounds using Monte-Carlo simulations for single integrator and curvature constrained systems and are able to validate the previous results presented by Karaman and Frazzoli [11] independently using the novel connections introduced in this paper.
\end{abstract}

\section{INTRODUCTION}

A very well studied problem in motion planning literature is finding dynamically feasible collision free trajectories for ground and aerial vehicles moving through unstructured environments, and there exists several state of the art approaches to solving this problem [13]. However, the success of these algorithms in practice depends on two key factors. Firstly, what is the maximum speed at which a collision free trajectory is guaranteed to exist with a high probability? If the robot violates this speed limit, there is a non-zero probability that it will encounter situations where a collision free trajectory does not exist, irrespective of the planning algorithm being used. Secondly, if the robot is operating below the speed limit, what is the minimum planning resolution of an algorithm such that it can compute a collision free trajectory. This controls the trade-off between time complexity and the success of the planning algorithm. The answer to both of these questions is fundamentally linked to the dynamics of the robot and the density of obstacles in the environment.

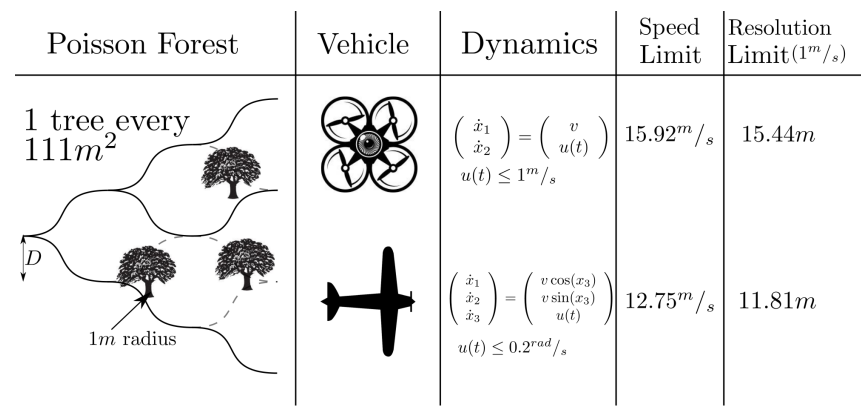

Fig. 1: Robot dynamics affect maximum speed and minimum planning resolution. The less conservative dynamics of the quadrotor allow for a higher speed as well as planning with a coarser resolution while still ensuring collision free motion.

Karaman and Frazzoli [11] solved the problem of the theoretical speed limit of a system with single integrator dynamics flying in a Poisson forest. They show that the system undergoes a phase transition - that above a critical speed there exists no infinite collision-free trajectory with probability 1 , while below this speed a path exists almost surely. By mapping the lattice obtained from the single integrator dynamics to a regular lattice, they use known results from discrete percolation theory $[3,8,19]$ to compute bounds on the speed. They [12] also show that the success of a planning algorithm for a system with nonlinear dynamics undergoes a phase transition using arguments based on k-independent bond percolation. However, they were unable to show how one may compute explicit bounds for such problems because of the lack of usable results in k-independent percolation theory.

In this paper, we analyze both problems under a single framework and provide expressions for the speed limit and resolution limit as a function of robot dynamics and obstacle density. The major milestones of our approach are

1) We show that solutions to both problems are equivalent to solving bond percolation on a directed asymmetric hexagonal lattice. Any variation in the problem, robot dynamics or obstacle density corresponds to different edge probabilities on the hexagonal lattice.

2) Since standard percolation theory lacks analysis of such a lattice, we map the hexagonal lattice to a discrete time Markov chain. Using results pertaining to the survival of the Markov chain as proved by Liggett [14], we compute 
the conditions leading to percolation of the lattice.

3) As a result, we provide a solution framework that takes as input the robot dynamics and the obstacle density and returns the speed and resolution limit. Our framework is not only able to recover the results in [11], but provide results for problems which [12] could not provide explicit answers to.

4) A key difference in our approach from that of [11, 12] is that we derive a parametric lattice - where the parameters depend explicitly on the various aspects of the problem, and the conditions of percolation are analytic equations involving the parameters. This allows us to solve a wide range of problems without having to analyze new lattices for new problems.

There has been an extensive amount of work in developing planning algorithms that perform a discrete search on a state lattice [5, 9, 10, 15-18]. Ascertaining the optimum resolution is an important problem because of its effect on real-time performance.

Our main contributions are (under certain assumptions on the dynamics and obstacle generating process) -

1) Proving a theoretical bound for the resolution of a state lattice that contains an infinite collision free trajectory with probability 1 , as a function of the dynamics of the robot.

2) Proving a theoretical bound for the speed limit of a robot as a function of its non-linear dynamics to ensure the existence of an infinite collision free trajectory. This is shown to be a special case of 1 .

This paper is organized as follows. In Section II, we formalize the two problems we wish to solve. In Section III, we establish an equivalence with directed asymmetric hexagonal lattice bond percolation and provide conditions for it. In Section IV, we provide theoretical bounds for the speed limit and lattice resolution and show examples in Section V. Finally, we conclude the paper in Section VI.

\section{Problem Definition}

\section{A. The Dynamics of the Robot}

Let $X \subseteq \mathbb{R}^{n}$ be the configuration space, $U \subseteq \mathbb{R}^{m}$ be the control space and $W \subseteq \mathbb{R}^{2}$ be the workspace of the robot. Let $T \in \mathbb{R}_{\geq 0}$. Let the configuration space trajectory be $x:[0, T] \rightarrow X$, control trajectory be $u:[0, T] \rightarrow U$ and workspace trajectory be $y:[0, T] \rightarrow W$. The trajectories are said to be dynamically feasible if they satisfy the following set of differential equations.

$$
\begin{aligned}
\dot{x}(t) & =f_{v}(x(t), u(t)) \\
y(t) & =g_{v}(x(t))
\end{aligned}
$$

where $f_{v}(.,$.$) and g_{v}($.$) are Lipschitz continuous in all vari-$ ables, as well as time invariant. The parameter $v$ is the workspace speed.

We make a restrictive set of assumptions about the dynamics of the robot in order to proceed with the analysis. The dynamics are parameterized explicitly as a function of the workspace speed $v$ as the analysis in this paper is restricted to constant speed regimes.

The workspace dimension is 2 . Let $y_{1}:[0, T] \rightarrow \mathbb{R}$ and $y_{2}:[0, T] \rightarrow \mathbb{R}$ be the first and second dimension of the workspace trajectory $y:[0, T] \rightarrow W$. For a workspace speed $v$, let $X_{0, v} \subset X$ be the forward flight configuration space regime and $u_{v} \subset U$ be the forward flight control space regime. This implies if the robot starts at $x(0) \in X_{0, v}$ and follows the dynamics (1) with $u(t) \in u_{v}$, then $x(t) \in X_{0, v}, \dot{y}_{1}(t)=v$ and $\dot{y}_{2}(t)=0$ for all $t \in \mathbb{R}_{\geq 0}$. In other words, the robot continues forward flight with constant velocity in this regime.

The dynamics also has a property of constrained lateral motion which is defined as follows. Let the robot starts at $x(0) \in X_{0, v}$ and let $t_{f}$ be the minimum time to reach a workspace position $y_{2}\left(t_{f}\right)>r$, where $r \in \mathbb{R}_{>0}$. The length of the corresponding workspace trajectory $\|y(t)\|$ increases monotonically with an increase in $v$. This assumption forces a notion of a speed limit at which the robot can no longer move laterally to avoid an obstacle of size $r$.

\section{B. The Stochastic Obstacle Field}

The environment is assumed to contain disc shaped obstacles in the plane to represent a planar forest. Let $(\Omega, \mathcal{F}, \mathbb{P})$ be a probability space where $\Omega$ is the sample space, $\mathcal{F}$ is the $\sigma$-algebra and $\mathbb{P}$ is the probability measure. Let $\Pi_{\rho}$ be the set of points generated by a homogeneous Poisson process with intensity $\rho$. The realizations of $\Pi_{\rho}$ are denoted by $\Pi_{\rho}(\omega)$, where $\omega \in \Omega$. Let $r \in \mathbb{R}$ be the radius of the obstacle. Thus the occupied workspace is

$$
W_{o b s}^{\rho, r}(\omega):=\bigcup_{l \in \Pi_{\rho}(\omega)} B(l, r)
$$

where $B(l, r)$ is a disc of radius $r$ centered at $l \in \mathbb{R}^{2}$. The free workspace is a random set $W_{f r e e}^{\rho, r}=W \backslash W_{o b s}^{\rho, r}$. We now define an infinite collision free trajectory

Definition 1: Given a Poisson forest of density $\rho$ and radius $r$, an infinite collision free trajectory is defined as a workspace trajectory $y(t)$ that

(i) makes progress towards new regions with probability 1

$$
\mathbb{P}\left(\left\{\lim \inf _{t \rightarrow \infty}\|y(t)\|=\infty\right\}\right)=1
$$

(ii) avoids collision with obstacles almost surely

$$
\mathbb{P}\left(\left\{y(t) \in W_{\text {free }}^{\rho, r}, \forall t \in \mathbb{R}_{\geq 0}\right\}\right)=1
$$

The homogenous poisson process is a simple instance of an ergodic marked point process [11], the properties of which allows us to proceed with the analysis. For arbitrary distributions, the spatial independence and invariance properties of the ergodic marked point process are not guaranteed to be true and our analysis will not hold. 


\section{Goal-directed Lattice Planner}

The motion planner used in this paper is a discrete graph search on a state lattice [18]. A state lattice is a graph $(V, E)$, where the vertices $V$ are discrete values of the state and the edges $E$ are dynamically feasible trajectories. The lattice in this paper is conservative and specifically designed for analysis purposes. The claim is that if an infinite collision free trajectory exists in this lattice, it exists in a more dense lattice that contains this as a subgraph.

To define the connectivity of the state lattice, we need to define a directed square lattice. Let $\mathbb{Z}$ denote the space of integers. Let $\mathbb{Z}_{+}^{2}$ be the positive orthant of the space of 2 dimensional vectors with integer elements. Consider a graph which has vertex set $\mathbb{Z}_{+}^{2}$ with element $z_{i}=\left(z_{i}^{1}, z_{i}^{2}\right) \in \mathbb{Z}_{+}^{2}$. There exists an edge between vertices $z_{i}, z_{j}$ whenever $z_{j}^{k}=$ $z_{i}^{k}+1$ for any $k \in\{1,2\}$ and $z_{1}^{k^{\prime}}=z_{2}^{k^{\prime}}$ for $k^{\prime} \neq k$. Then this graph is a directed square lattice in $\mathbb{Z}_{+}^{2}$ as shown in Fig. $2 b$.

Definition 2: For a desired forward speed $v$, and obstacle radius $r$, the state lattice that we consider in this paper has the following properties

(i) $V \subset X_{0, v}$ is a countable set of states that are all in the forward flight regime such that the workspace resolution is $D$.

(ii) $E \subset V \times V$ is a set of edges that are dynamically feasible trajectories $y(t)$ satisfying boundary value conditions such that $y(t) \in W_{\text {free }}^{\rho, r}$.

(iii) There exists a labeling function $\phi: V \rightarrow \mathbb{Z}_{+}^{2}$ such that for all $v, v^{\prime} \in V$ with $\left(v, v^{\prime}\right) \in E$, there exists an edge from $\phi(v)$ to $\phi\left(v^{\prime}\right)$ in the directed square lattice on $\mathbb{Z}_{+}^{2}$. This implies the state lattice is isomorphic to the directed square lattice.

(iv) For any 4-tuple $v_{1}, v_{2}, v_{3}, v_{4} \in V$ such that $\phi\left(v_{1}\right), \phi\left(v_{2}\right), \phi\left(v_{3}\right), \phi\left(v_{4}\right)$ are all distinct, the edges (if they exist $) y^{12}(t)=\left(v_{1}, v_{2}\right)$ and $y^{34}(t)=\left(v_{3}, v_{4}\right)$ satisfy the relation $\min _{s, t}\left\|y^{12}(s)-y^{34}(t)\right\|>2 r$. This implies edges not sharing a common vertex cannot be invalidated by the same obstacle.

Fig. 2a shows an example of the lattice.

\section{Problem Formulation}

Problem 1: Given the dynamics of the robot described in (1) and a specified actuator limit $u(t) \leq u_{\max }, \forall t \in \mathbb{R}_{\geq 0}$, find the speed limit $v$ such that there exists an infinite collision free trajectory $y(t)$.

Problem 2: Given the dynamics of the robot described by (1), an operating forward speed $v$ and a specified actuator limit $u(t) \leq u_{\max }, \forall t \in \mathbb{R}_{\geq 0}$, find the resolution limit $D$ of a state lattice defined in Definition 2, such that it contains an infinite collision free path $y(t)$.

\section{E. Key Assumptions}

We collect all the key assumptions that we make in this paper in order to apply our method of analysis

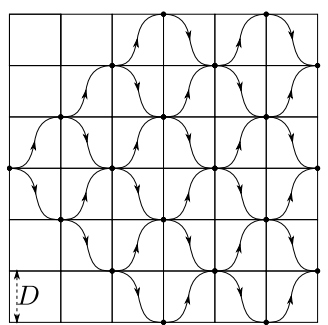

(a)

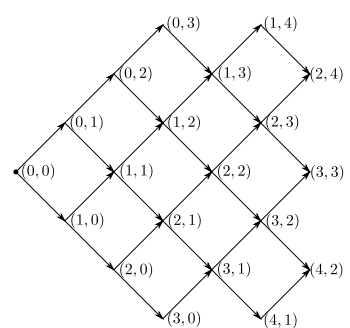

(b)
Fig. 2: Isomorphism of the state lattice with a directed square lattice. (a) The state lattice in Definition 2 with resolution $D$. (b) A directed square lattice in $\mathbb{Z}_{+}^{2}$ rotated by $-\frac{\pi}{4}$ to emphasize the isomorphism with the state lattice. The labeling function $\phi(v)$ returns the coordinates of the corresponding vertex in the directed square lattice.

1) Dynamics: The robot follows the dynamics in (1), the workspace is restricted to $2 \mathrm{D}$ and the dynamics have the property of constrained lateral motion.

2) Obstacle: The obstacles are of fixed radius $r$ generated by a homogenous Poisson process.

3) Lattice: The state lattice used for motion planning is specified in Definition 2.

4) Problem: We will compute a lower bound to speed $v$ and resolution $D$ at which an infinite collision free trajectory exists almost surely. This lower bound will still hold if the robot is not restricted to the proposed lattice (although the bounds will be significantly less tight).

Note that among the various assumptions, the assumption about the obstacle generating process restricts the applicability of this work. Violation of the homogenous poisson process could result in situations where a phase transition will not occur rendering the objective of this paper to invalid. This is an area that requires further research.

\section{Discrete Percolation on a Hexagonal lattice}

In this section we will use the fact that the structure of the problems defined in the previous section is equivalent to the existence of an infinite cluster of connected vertices on the state lattice. Using the theory of discrete percolation, the conditions required for such an event can be analytically derived for certain regular lattices. By finding a suitable equivalence between the state lattice and a regular lattice, we will provide answers to Problem 1 and 2.

\section{A. Theory of Discrete Percolation}

The directed lattice bond percolation model is as follows. Given a directed lattice, an edge is declared open with probability $p$, independent of every other edge and closed otherwise. An open path is a sequence $\left(z_{0}, z_{1}, \ldots, z_{k}\right)$ of vertices such that an open edge exists between $z_{i-1}$ and $z_{i}$ for all $i \in\{1,2, \ldots, k\}$. An open cluster is a maximal set of vertices that are connected with open paths. An infinite open cluster is an open cluster that has infinite vertices. Percolation is said to occur for a value of $p$ if there exists an infinite 
open cluster. The following theorem as stated by Durrett [7] summarizes this

Theorem 1. As the value of $p$ increases from 0 to 1 , for a critical probability $p_{\text {crit }}$, if $p<p_{\text {crit }}$ then almost surely there is no infinite open cluster. If $p>p_{\text {crit }}$ then there exists a unique infinite open cluster.

Proof: See Durrett [7] (Equations (7.6) and (12.1))

Results for $p_{\text {crit }}$ exist for a wide range of lattices such as square, triangle and hexagonal. In most cases $p_{\text {crit }}$ is not known exactly, but is bounded above and below. In order to transform Problem 1 and 2 to directed percolation on a lattice for which analysis is possible, a suitable equivalence must be established. The equivalence must be made such that edges on the transformed lattice have independent probability of being closed or open. It is important to note that we are interested in the upper bound of $p_{\text {crit }}$ because the problem requires computing conditions where a solution is guaranteed to exist.

\section{B. Equivalence to a Hexagonal Lattice}

We will now establish the equivalence of the state lattice in Definition 2 with directed asymmetric hexagonal lattice percolation.

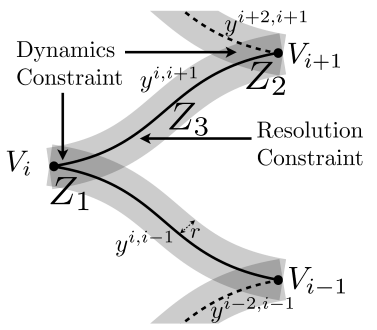

(a)

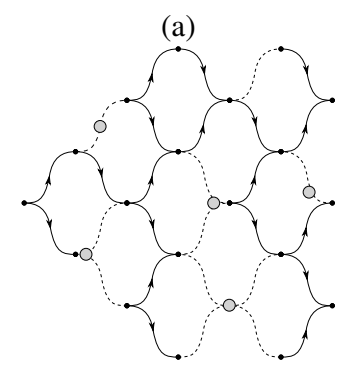

(c)

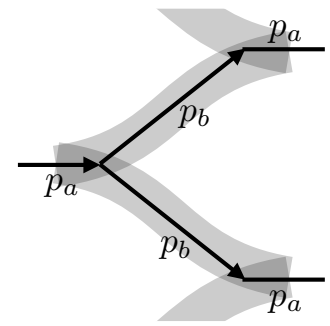

(b)

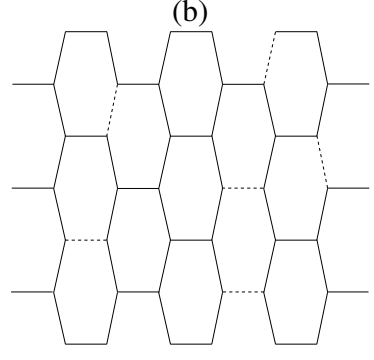

(d)
Fig. 3: Equivalence of state lattice with a directed asymmetric hexagonal lattice. (a) Adjacent vertices in a state lattice along with the swaths of edges. Different zones correspond to regions of overlap of the swath. (b) An equivalent hexagonal lattice which maps zones of the swath to edges on the lattice (c) The open cluster of a state lattice in the presence of a Poisson obstacle field (d) The open cluster of the hexagonal lattice that is equivalent to the state lattice.

Fig. 3c illustrates Problem 2, where percolation on the state lattice would ensure an infinite collision free trajectory. However, the state lattice does not satisfy the edge independence property as edges sharing a common vertex are within $r$ distance of each other and can be closed simultaneously. Thus we need the following theorem.

Theorem 2. Given a state lattice satisfying Definition 2, there exists a directed asymmetric hexagonal lattice with independent edge collision probability such that the state lattice contains an infinite open cluster iff the hexagonal lattice contains an infinite open cluster.

Proof: Since it is assumed that the dynamics of the robot (1) is translation invariant and the obstacle generation process is ergodic, the analysis for dependence of incoming and outgoing edges for a vertex holds for any other vertex in the lattice. Fig. 3a focuses on a set of adjacent vertices of the state lattice. Let $y^{i, i+1}(t)$ be the workspace trajectory corresponding to the lattice edge joining the vertex $V_{i}$ at time $t=0$ and the vertex $V_{i+1}$ at time $t=\tau$. The swath of the segment, $S\left(y^{i, i+1}\right)$ is defined by the union of the area swept out by a disc of radius $r$ following $y^{i, i+1}$

$$
S\left(y^{i, i+1}\right)=\bigcup_{s \in[0, \tau]} B\left(y^{i, i+1}(s), r\right)
$$

The swath consists of 3 zones

1) $Z_{1}=S\left(y^{i, i+1}\right) \cap S\left(y^{i, i-1}\right)$

2) $Z_{2}=S\left(y^{i, i+1}\right) \cap S\left(y^{i+2, i+1}\right)$

3) $Z_{3}=S\left(y^{i, i+1}\right)-Z_{1}-Z_{2}$

$Z_{1}$ and $Z_{2}$ are strongly coupled to the dynamics constraints that couple adjacent edges on the lattice. An obstacle appearing in these zones would invalidate both edges. $Z_{3}$ exists because of a resolution constraint that prevents sampling in a dense manner. An obstacle in this zone would only invalidate the corresponding edge. Also note that from the constrained lateral motion property of the dynamics (Section II-A), $Z_{1}+Z_{2}$ increase with speed $v$ - adjacent edges cover a larger distance before the swaths no longer overlap.

By combining $Z_{1}$ from one segment and $Z_{2}$ from its parent segment, we map this combined zone to a horizontal bond $a$. The zone $Z_{3}$ is mapped to a connecting bond $b$. Thus the swath zones are mapped to bonds in a hexagonal lattice as shown in Fig. 3b. An obstacle appearing in zone $Z_{1}$ or $Z_{2}$ is equivalent to invalidating bond $a$. Similarly an obstacle appearing in zone $Z_{3}$ is equivalent to invalidating bond $b$. The probabilities of $a$ and $b$ being closed or open are independent of each other. Thus percolation on this directed hexagonal lattice is equivalent to percolation on the original state lattice. As a result an equivalence has been established.

Let the probability of a bond being open be $p_{a}$ and $p_{b}$ respectively for bonds $a$ and $b$. Under the assumption of an ergodic distribution of obstacles, $p_{a}$ and $p_{b}$ are independent of vertex $i$ and time $t$ and can be computed as

$$
\begin{aligned}
p_{a} & =e^{-\rho\left(Z_{1}+Z_{2}\right)} \\
p_{b} & =e^{-\rho Z_{3}}
\end{aligned}
$$

Since $p_{a}$ and $p_{b}$ need not be equal, the model is that of directed asymmetric hexagonal lattice bond percolation. Conditions leading to percolation on this lattice leads to the existence of infinite collision free trajectories. 


\section{Directed Asymmetric Hexagonal Lattice Bond Percolation}

Directed bond percolation in general is a much harder problem than undirected as described by Durrett [7]. Moreover, it has been shown that finding the lower bound is much simpler than the upper bound. For example, an upper bound on the directed square lattice has been provided by Durrett [7] and later improved by Balister et al. [1, 2]. However, these results are difficult to apply to directed asymmetric hexagonal lattice bond percolation due to the violation of symmetry. An approach is required that can offer a tight upper bound, yet is parameterized in terms of $p_{a}$ and $p_{b}$.

Liggett [14] offered such a tool that can compute upper bounds for the discrete percolation problem using a completely different approach in comparison to percolation literature. He linked the existence of an infinite open cluster to survival of a discrete time Markov chain.

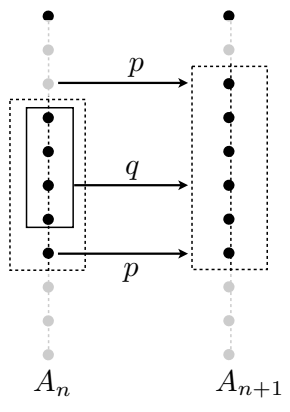

(a)

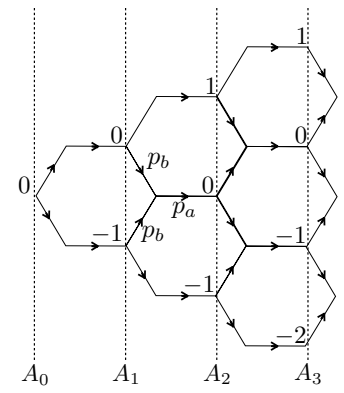

(b)
Fig. 4: (a) Illustration of the Markov chain defined in (3) as a collection of subsets of integers on the number line. At time slice $n$, the set $A_{n}$ is shown by elements in the rectangle. The transition probabilities of elements with a neighbour in $A_{n}$ is shown as $q$ while elements with one neighbour is shown as $p$. (b) The hexagonal lattice is represented as the Markov chain. Each vertex is assigned an integer to map to the integer line. A vertex is contained in $A_{n}$ if there exists an open path from the origin to it.

The model Liggett examined was the discrete time Markov chain $A_{n}$ on the collection of finite subsets of integers. This is illustrated in Fig. 4a. The two parameters in the chain are $0 \leq$ $p \leq q \leq 1$. Given $A_{n}$, the events $x \in A_{n+1}$ are conditionally independent and

$$
P\left(x \in A_{n+1} \mid A_{n}\right)= \begin{cases}q & \text { if }\left|A_{n} \cap\{x, x+1\}\right|=2 \\ p & \text { if }\left|A_{n} \cap\{x, x+1\}\right|=1 \\ 0 & \text { if }\left|A_{n} \cap\{x, x+1\}\right|=0\end{cases}
$$

Liggett then proved the following result.

Theorem 3. If the parameters $p$ and $q$ satisfy the inequalities

$$
\frac{1}{2}<p \leq 1, \quad 4 p(1-p) \leq q \leq 1
$$

then the Markov chain $A_{n}$ satisfies $\mathbb{P}\left(\forall n: A_{n} \neq \emptyset\right)>0$
Proof: For brevity, we omit the proof in this paper and refer the reader to a detailed discussion in [4] and [14] for the original proof.

For intuition and references regarding the proof for the above result, the reader is directed to the Appendix section.

The result can be applied to derive an upper bound for the critical probability for the directed asymmetric hexagonal lattice bond percolation. Fig. 4b illustrates the mapping of the hexagonal lattice to a Markov chain. $A_{n}$ is the set of vertices at time $n$ that can be reached starting from the origin $A_{0}$. To structure the lattice as the Markov chain in (3), the vertices are assigned an integer $x$. For columns $A_{2 i}$, the origin $x=0$ is at the same $y$ coordinate as the starting point. For columns $A_{2 i+1}$, the origin is shifted up by one lattice resolution. The corresponding transition probabilities are

$$
\begin{aligned}
p & =p_{a} p_{b} \\
q & =2 p_{a} p_{b}-p_{a} p_{b}^{2}
\end{aligned}
$$

According to Theorem 3 , the conditions on $p_{a}$ and $p_{b}$ for percolation, $C\left(p_{a}, p_{b}\right) \leq 0$, are as follows

$$
\begin{aligned}
0 \leq p_{a} & \leq 1 \\
0 \leq p_{b} & \leq 1 \\
\frac{1}{2}<p_{a} p_{b} & \leq 1 \\
p_{a} p_{b}\left(2-p_{b}\right) & \leq 1 \\
p_{b}-4 p_{a} p_{b}+2 & \leq 0
\end{aligned}
$$

As long as the inequalities $C\left(p_{a}, p_{b}\right) \leq 0$ in (5) are true, the lattice will almost surely have an infinite open cluster. In the following section, we will show how parameters that dictate the structure of the state lattice such as dynamics of the robot and resolution of the lattice map into $p_{a}$ and $p_{b}$.

\section{ANALYSIS}

In this section, we use the set of constraints $C\left(p_{a}, p_{b}\right) \leq 0$ stated in (5) to provide analytical expressions for the solutions of Problem 1 and 2.

\section{A. Problem 1: Speed limit with unconstrained planning reso- lution}

We will now show that a solution to Problem 1 can be arrived at using the results in Section III. Note that finding the true speed limit analytically lies beyond the scope of the mathematical tools that have been referred to. Instead we will aim to find a lower bound of the speed limit. We do this by enforcing the solution to lie on a lattice satisfying Definition 2.

To obtain the maximum velocity for which an infinite collision free trajectory exists, we wish to solve the following optimization problem

$$
\begin{aligned}
& \max _{p_{a}, p_{b}} v \quad \text { s.t } C\left(p_{a}, p_{b}\right) \leq 0 \\
& =\max _{p_{a}, p_{b}} \quad Z_{1}+Z_{2} \quad \text { s.t } \quad C\left(p_{a}, p_{b}\right) \leq 0 \\
& =\min _{p_{a}, p_{b}} p_{a} \quad \text { s.t } \quad C\left(p_{a}, p_{b}\right) \leq 0
\end{aligned}
$$




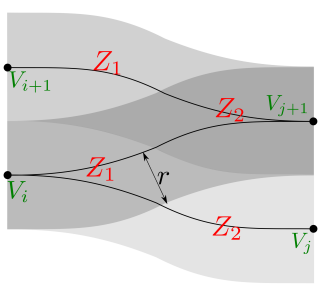

(a)

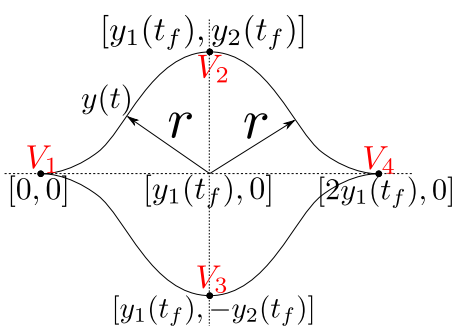

(b)
Fig. 5: State lattice at the speed limit (a) The speed limit is achieved by the state lattice by forcing $Z_{3}=0$. This is intuitive because $Z_{3}$ is unconstrained and percolation probability increases with decreasing $Z_{3}$. (b) The minimum resolution of the state lattice is such that edges not sharing a common vertex have independent collision probability. Thus edges $\left(V_{1}, V_{2}\right)$ and $\left(V_{2}, V_{4}\right)$ should be farther than $r$ from the origin.

The solution to this is

$$
p_{a}=\frac{3}{4}, \quad p_{b}=1
$$

This corresponds to $Z_{3}=0$ as shown in Fig. 5a. It is interesting to note that this is the percolation threshold for directed site percolation on a square lattice which is what the problem has reduced to.

Using (6) in (4)

$$
\begin{aligned}
e^{-\rho\left(Z_{1}+Z_{2}\right)} & \geq \frac{3}{4} \\
Z_{1}+Z_{2} & \leq \frac{1}{\rho} \log \frac{4}{3}
\end{aligned}
$$

In order to solve for the motion primitives of this lattice for a particular velocity, it is required that $Z_{1}+Z_{2}$ is minimized so that $p_{a}$ is above the threshold computed in (6). To minimize the area for a fixed velocity, a time optimal trajectory is required to connect adjacent vertices of the state lattice.

However, the resolution of the lattice is still to be ascertained. This is done by enforcing edges that do not share a common vertex to have independent collision probability. In Fig. 5 b, $\left(V_{1}, V_{2}\right)$ should be $2 r$ distance from $\left(V_{3}, V_{4}\right)$. By symmetry, this implies

$$
\left\|y(t)-\left[\begin{array}{ll}
y_{1}\left(t_{f}\right) & 0
\end{array}\right]^{T}\right\| \geq r
$$

Also $\left(V_{1}, V_{3}\right)$ should be $2 r$ distance from $\left(V_{2}, V_{4}\right)$. By using symmetry, this is

$$
\left\|y(t)-\left[\begin{array}{ll}
0 & y_{2}\left(t_{f}\right)
\end{array}\right]^{T}\right\| \geq r
$$

Thus the time optimal control problem subject to the velocity constraints, boundary constraints and edge separation constraints (8) and (9) is as follows

$$
\begin{array}{ll}
\underset{x(t), u(t), y(t)}{\operatorname{minimize}} & t_{f} \\
\text { subject to } & \dot{x}(t)=f(x(t), u(t)) \\
& y(t)=g(x(t)) \\
& y(0)=\left[\begin{array}{ll}
0 & 0
\end{array}\right]^{T} \\
& x(0) \in X_{0, v} \\
& x\left(t_{f}\right) \in X_{0, v} \\
& \left\|y(t)-\left[\begin{array}{ll}
y_{1}\left(t_{f}\right) & 0
\end{array}\right]^{T}\right\| \geq r \\
& \left\|y(t)-\left[\begin{array}{ll}
0 & y_{2}\left(t_{f}\right)
\end{array}\right]^{T}\right\| \geq r
\end{array}
$$

From Fig. 5a we can see that $Z_{1}+Z_{2}$ is actually the swath of the path.

$$
Z_{1}+Z_{2}=\left|\bigcup_{s \in\left[0, t_{f}\right]} B(y(s), r)\right|
$$

Since the solution of the optimization $y(t)$ is a function of speed $v$, from (11) we have $Z_{1}+Z_{2}=\mu(v)$. Substituting this in (7), we have

$$
\begin{aligned}
\mu(v) & \leq \frac{1}{\rho} \log \frac{4}{3} \\
v & \leq \mu^{-1}\left(\frac{1}{\rho} \log \frac{4}{3}\right)
\end{aligned}
$$

Thus (12) gives a bound on $v_{c r i t}$, a speed limit at which there exists an infinite collision free trajectory.

\section{B. Problem 2: Planning resolution limit in sub-critical speed regime}

If the robot is operating in the sub-critical speed regime $v<v_{\text {crit }}$, and is required to plan at a resolution $D$, the zone $Z_{3} \neq 0$. The objective then is to maximize the resolution $D$ subject to the constraint that an infinite collision free trajectory exists. This can be formulated as the following optimization problem.

$$
\max _{p_{a}, p_{b}} D \quad \text { s.t } \quad C\left(p_{a}, p_{b}\right) \leq 0
$$

The lattice edge is defined by time optimal trajectory that connects the adjacent vertices. This is represented as follows

$$
\begin{array}{ll}
\underset{x(t), u(t), y(t)}{\operatorname{minimize}} & t_{f} \\
\text { subject to } & \dot{x}(t)=f_{v}(x(t), u(t)) \\
& y(t)=g_{v}(x(t)) \\
& y(0)=\left[\begin{array}{ll}
0 & 0
\end{array}\right]^{T} \\
& y\left(t_{f}\right)=\left[\begin{array}{ll}
D & D
\end{array}\right]^{T} \\
& x(0) \in X_{0, v} \\
& x\left(t_{f}\right) \in X_{0, v} \\
& \left\|y(t)-\left[\begin{array}{ll}
y_{1}\left(t_{f}\right) & 0
\end{array}\right]^{T}\right\| \geq r \\
& \left\|y(t)-\left[\begin{array}{ll}
0 & y_{2}\left(t_{f}\right)
\end{array}\right]^{T}\right\| \geq r
\end{array}
$$


Having computed $y(t)$, and denoting $\tilde{y}(t)$ as its reflection about the $\mathrm{x}$-axis, the zones can be computed as follows

$$
\begin{aligned}
& Z_{1}+Z_{2}=\mid \bigcup_{s \in\left[0, t_{f}\right]} B(y(s), r) \cap(B(\tilde{y}(s), r) \cup \\
& \left.B\left(\left[\begin{array}{ll}
0 & D
\end{array}\right]^{T}+\tilde{y}(s), r\right)\right) \\
& Z_{3}=\left|\bigcup_{s \in\left[0, t_{f}\right]} B(y(s), r)-\left(Z_{1}+Z_{2}\right)\right|
\end{aligned}
$$

As the zones $Z_{1}(D), Z_{2}(D), Z_{3}(D)$ are a function of the resolution $D$, substituting in (2) we have $p_{a}(D), p_{b}(D)$. This is substituted in (5) to get $C\left(p_{a}(D), p_{b}(D) \leq 0\right.$ in order to obtain $D_{\text {crit }}$. $D_{\text {crit }}$ corresponds to the resolution at which infinite collision free path can be guaranteed. (10) and (13) are generally non-convex optimization problems. The example dynamics in this paper have analytic solutions, however in general this has to be solved numerically by collocation methods.

\section{RESUlts}

In this section we present results for both problems with different robot dynamics. Monte-Carlo simulations are performed to compare experimental data with theoretical bounds. As the bounds are for infinite open clusters, the simulations, which are done for a large finite forest, reflect a lower bound of the true collision probability. In all of these experiments, the size of the forest is $L=500 \mathrm{~m}$.

\section{A. Problem 1: Speed limit with unconstrained planning reso- lution}

1) Single Integrator Dynamics: We first consider the simple linear dynamics used by Karaman and Frazzoli [11]. The configuration space is $X=[x, y]^{T}$. The speed of the robot is $v$ and the control input is lateral speed. The dynamics of the robot is

$$
\dot{x}(t)=\left(\begin{array}{c}
\dot{x}_{1} \\
\dot{x}_{2}
\end{array}\right)=\left(\begin{array}{c}
v \\
u(t)
\end{array}\right), y(t)=x(t)
$$

where $|u(t)| \leq u_{\max }$.

Let this form a lattice of angle $\alpha$ at speed $v$ such that $\tan \frac{\alpha}{2}=\frac{u_{\max }}{v}$. From (10), we have $y\left(t_{f}\right)=\frac{r}{\cos \frac{\alpha}{2}}$ as shown in Fig. 7a. Thus $Z_{1}+Z_{2}$ from (11) is

$$
\begin{aligned}
Z_{1}+Z_{2} & =2 r \sqrt{\left(\frac{r}{\sin \frac{\alpha}{2}}\right)^{2}+\left(\frac{r}{\cos \frac{\alpha}{2}}\right)^{2}} \\
& =\frac{4 r^{2}}{\sin \alpha}
\end{aligned}
$$

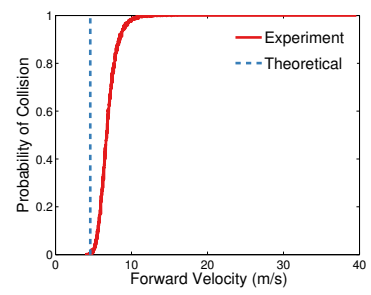

(a)

(c)

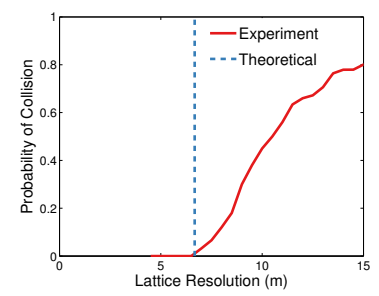

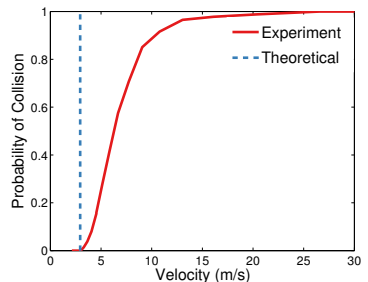

(b)

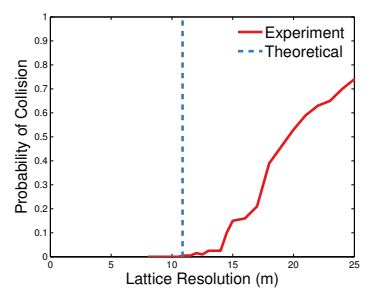

(d)
Fig. 6: Phase transition diagrams for different experiments obtained by Monte-Carlo simulations of Poisson forest of density $\rho$ and radius $r$. The experiments are performed on the lattice described in the paper. The red line shows the probability of collision against the parameter being varied. The blue dashed line is the theoretical bound below which probability of collision is 0 . (a) Single integrator dynamics with $u_{\max }=1 \mathrm{~m} / \mathrm{s}$, forest parameters $\rho=0.03, r=1 \mathrm{~m}$, 1000 trials and theoretical bound $v_{\text {crit }}=4.58 \mathrm{~m} / \mathrm{s}$ (b) Curvature constrained dynamics with $\omega_{\max }=0.52 \mathrm{rad} / \mathrm{s}$, forest parameters $\rho=0.03, r=1 \mathrm{~m}, 1000$ trials and theoretical bound $v_{\text {crit }}=2.94 \mathrm{~m} / \mathrm{s}$ (c) Single integrator dynamics with $u_{\max }=1 \mathrm{~m} / \mathrm{s}, v=1 \mathrm{~m} / \mathrm{s}$, forest parameters $\rho=0.02, r=1 \mathrm{~m}$, 500 trials and theoretical bound $D_{\text {crit }}=6.67 \mathrm{~m}$ (d) Curvature constrained dynamics with $\omega_{\max }=0.52 \mathrm{rad} / \mathrm{s}, v=1.56 \mathrm{~m} / \mathrm{s}$, forest parameters $\rho=0.01, r=1 \mathrm{~m}, 500$ trials and theoretical bound $D_{\text {crit }}=10.87 \mathrm{~m}$.

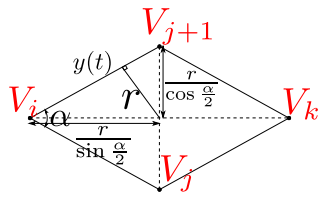

(a)

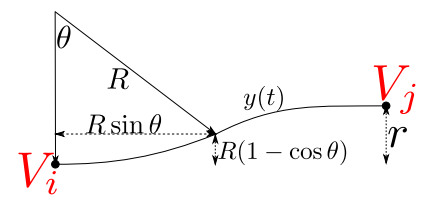

(b)
Fig. 7: (a) The lattice for single integrator dynamics operating at speed limit has angle $\alpha$. (b) The bang-bang motion for curvature constrained dynamics of radius $R$ turning $\theta$ and $-\theta$.

Using (16) in (7), we have

$$
\begin{aligned}
\frac{4 r^{2}}{\sin \alpha} & \leq \frac{1}{\rho} \log \frac{4}{3} \\
\alpha & \geq \sin ^{-1} \frac{4 \rho r^{2}}{\log \frac{4}{3}} \\
v & \leq \frac{u_{\max }}{\tan \left(\frac{1}{2} \sin ^{-1} \frac{4 \rho r^{2}}{\log \frac{4}{3}}\right)}
\end{aligned}
$$

Solving (17) for $u_{\max }=1, \rho=0.03, r=1 \mathrm{~m}$ we get 
$v_{\text {crit }}=4.58 \mathrm{~m} / \mathrm{s}$ which is shown in comparison with empirical result in Fig. 6a. Note that Karaman and Frazzoli [11] reached the same result through a different formulation.

2) Curvature Constrained Dynamics: To demonstrate results for non-linear dynamics, we consider a curvature constrained robot. The configuration space is $X=[x, y, \psi]^{T}$. The speed of the robot is $v$ and the control input is the angular speed. Then the dynamics of the robot is

$$
\dot{x}(t)=\left(\begin{array}{c}
\dot{x}_{1} \\
\dot{x}_{2} \\
\dot{x}_{3}
\end{array}\right)=\left(\begin{array}{c}
v \cos \left(x_{3}\right) \\
v \sin \left(x_{3}\right) \\
u(t)
\end{array}\right), y(t)=\left(\begin{array}{c}
x_{1} \\
x_{2}
\end{array}\right)
$$

where $|u(t)| \leq \omega_{\max }$.

Let $R=\frac{v}{\omega_{\max }}$ be the minimum radius of curvature. For the derivation below, we assume that $R>r$. The time optimal trajectory for this class of dynamics has been solved by Dubins [6]. For this case, the curve reaches the end point by a bangbang motion

$$
u(t)= \begin{cases}\omega_{\max } & 0 \leq t \leq \frac{t_{f}}{2} \\ -\omega_{\max } & \frac{t_{f}}{2}<t \leq t_{f}\end{cases}
$$

From (19), let $\theta=\frac{\omega_{\max } t_{f}}{2}$ be the change of angle as shown in Fig. 7b. From (8) and (9), we have $\theta=\cos ^{-1}\left(1-\frac{r}{2 R}\right)$. From (11), $Z_{1}+Z_{2}$ is

$$
\begin{aligned}
Z_{1}+Z_{2} & =4 r R \theta \\
& =4 r R \cos ^{-1}\left(1-\frac{r}{2 R}\right)
\end{aligned}
$$

Substituting (20) in (7), we have

$$
4 r R \cos ^{-1}\left(1-\frac{r}{2 R}\right) \leq \frac{1}{\rho} \log \frac{4}{3}
$$

Solving (21) for $r=1 \mathrm{~m}$ and $\rho=0.03$, we have $R=$ $5.66 \mathrm{~m}$. For $\omega_{\max }=0.52^{\mathrm{rad}} / \mathrm{s}$, we get a $v_{\text {crit }}=2.94 \mathrm{~m} / \mathrm{s}$ which is shown in comparison with empirical data in Fig. 6b. Note that this is much smaller than the single integrator speed limit.

B. Problem 2: Planning resolution limit in sub-critical speed regime

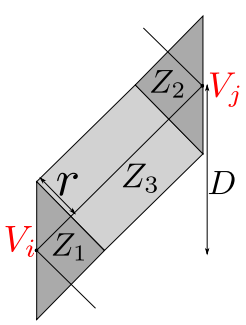

(a)

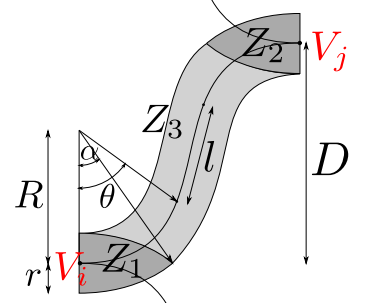

(b)
Fig. 8: (a) Lattice of resolution $D$ with angle $\alpha=\frac{\pi}{2}$ for single integrator dynamics. (b) Lattice of resolution $D$ for curvature constrained dynamics of radius $R$ where the edge turns by $\theta$, travels $l$ and turns back by $-\theta$. $\alpha$ is the angle at which overlapping swaths become independent.
1) Single Integrator Dynamics: Let the robot have the dynamics described in (15). Let $v=1 \mathrm{~m} / \mathrm{s}, u_{\max }=1 \mathrm{~m} / \mathrm{s}$. From (14), we have

$$
\begin{aligned}
Z_{1}+Z_{2} & =4 r^{2} \\
Z_{3} & =2 r(2 \sqrt{2} D)-4 r^{2}
\end{aligned}
$$

Subject to constraints in (5), and maximizing $D$ we get for $r=1 \mathrm{~m}$ and $\rho=0.02$, a bound of $D_{\text {crit }}=6.67 \mathrm{~m}$ which is compared to emprical results in Fig. 6c.

2) Curvature Constrained Dynamics: Let the robot have the dynamics described in (18). For the purposes of this example, we assume that the resolution $D>2 R$ such that the optimal path $y(t)$ on solving (13) is

$$
u(t)= \begin{cases}\omega_{\max } & 0 \leq t \leq t_{1} \\ 0 & t_{1}<t \leq t_{f}-t_{1} \\ -\omega_{\max } & t_{f}-t_{1}<t \leq t_{f}\end{cases}
$$

Let $\theta=\omega_{\max } t_{1}$ and $l=v\left(t_{f}-2 t_{1}\right)$. Then the following can be derived geometrically [6]

$$
\begin{aligned}
& l=\sqrt{2 D(D-2 R)} \\
& \theta=2 \tan ^{-1}\left(\frac{l-D}{D-4 R}\right)
\end{aligned}
$$

Fig. $8 \mathrm{~b}$ shows the angle $\alpha$ at which adjacent swaths decouple. We assume for this example $\alpha \leq \theta$. Then $\alpha=$ $\cos ^{-1}\left(1-\frac{r}{R}\right)$. From (14) we have

$$
\begin{aligned}
Z_{1}+Z_{2} & =2(R+r)^{2}\left(\alpha-\frac{1}{2} \sin (2 \alpha)\right) \\
Z_{3} & =2 r(2 R \theta+l)-Z_{1}-Z_{2}
\end{aligned}
$$

Subject to constraints in (5), and maximizing $D$ we get for $v=1.56 \mathrm{~m} / \mathrm{s}, \omega_{\max }=0.52^{\mathrm{rad}} / \mathrm{s}, r=1 \mathrm{~m}, \rho=0.01$, the critical resolution $D_{\text {crit }}=10.87 \mathrm{~m}$ which is compared with empirical results in Fig. 6d.

\section{CONCLUSION}

In this paper, we analyzed two problems related to motion planning for robots with nonlinear dynamics in homogeneous Poisson forests. First, we provided a bound for the speed limit of a robot as a function of its dynamics that ensures infinite collision free flight. Secondly, we provided a bound for the state lattice resolution as a function of the dynamics such that an infinite collision free solution is contained in the lattice. We proposed a novel way of drawing equivalence with a directed asymmetric hexagonal lattice and obtained bounds by mapping the lattice to a Markov chain and solving for its survivability.

\section{ACKNOWLEDGMENTS}

Drew Bagnell acknowledges the support of ONR MURI grant N00014-09-1-1051 "Provably-Stable Vision-Based Control of High-Speed Flight through Forests and Urban Environments". 


\section{REFERENCES}

[1] Paul Balister, Bela Bollobas, and Alan Stacey. Upper bounds for the critical probability of oriented percolation in two dimensions. Proceedings of the Royal Society of London. Series A: Mathematical and Physical Sciences, 440(1908):201-220, 1993.

[2] Paul Balister, Béla Bollobás, and Alan Stacey. Improved upper bounds for the critical probability of oriented percolation in two dimensions. Random Structures \& Algorithms, 5(4):573-589, 1994.

[3] Bela Bollobas and Oliver Riordan. Percolation. Cambridge University Press, 2006.

[4] Sanjiban Choudhury. Lower and upper bounds for the survival of infinite absorbing Markov chains. Technical Report CMU-RI-TR-05-04, Robotics Institute, Pittsburgh, PA, January 2015.

[5] Dmitri Dolgov, Sebastian Thrun, Michael Montemerlo, and James Diebel. Path planning for autonomous vehicles in unknown semi-structured environments. The International Journal of Robotics Research, 29(5):485501, 2010.

[6] Lester E Dubins. On curves of minimal length with a constraint on average curvature, and with prescribed initial and terminal positions and tangents. American Journal of mathematics, pages 497-516, 1957.

[7] Richard Durrett. Oriented percolation in two dimensions. The Annals of Probability, pages 999-1040, 1984.

[8] Geoffrey Grimmett. What is Percolation? Springer, 1999.

[9] Lionel Heng, Lorenz Meier, Petri Tanskanen, Friedrich Fraundorfer, and Marc Pollefeys. Autonomous obstacle avoidance and maneuvering on a vision-guided MAV using on-board processing. In Robotics and automation (ICRA), 2011 IEEE international conference on, pages 2472-2477. IEEE, 2011.

[10] Myung Hwangbo, James Kuffner, and Takeo Kanade. Efficient two-phase $3 \mathrm{~d}$ motion planning for small fixedwing uavs. In Robotics and Automation, 2007 IEEE International Conference on, pages 1035-1041. IEEE, 2007.

[11] Sertac Karaman and Emilio Frazzoli. High-speed flight in an ergodic forest. In Robotics and Automation (ICRA), 2012 IEEE International Conference on, pages 28992906. IEEE, 2012.

[12] Sertac Karaman and Emilio Frazzoli. High-speed motion with limited sensing range in a poisson forest. In $C D C$, pages 3735-3740, 2012.

[13] Steven M LaValle. Planning algorithms. Cambridge university press, 2006.

[14] Thomas M Liggett. Survival of discrete time growth models, with applications to oriented percolation. The Annals of Applied Probability, pages 613-636, 1995.

[15] Maxim Likhachev and Dave Ferguson. Planning long dynamically feasible maneuvers for autonomous vehicles. The International Journal of Robotics Research, 28(8): 933-945, 2009.
[16] Stephen R Lindemann and Steven M LaValle. Multiresolution approach for motion planning under differential constraints. In Robotics and Automation, 2006. ICRA 2006. Proceedings 2006 IEEE International Conference on, pages 139-144. IEEE, 2006.

[17] Brian MacAllister, Jonathan Butzke, Alex Kushleyev, Harsh Pandey, and Maxim Likhachev. Path planning for non-circular micro aerial vehicles in constrained environments. In Robotics and Automation (ICRA), 2013 IEEE International Conference on, pages 3933-3940. IEEE, 2013.

[18] Mihail Pivtoraiko, Ross A Knepper, and Alonzo Kelly. Differentially constrained mobile robot motion planning in state lattices. Journal of Field Robotics, 26(3):308333, 2009.

[19] M Sahini and M Sahimi. Applications of percolation theory. CRC Press, 1994. 